“(C) 2016 IEEE. Personal use of this material is permitted. Permission from IEEE must be obtained for all other uses, in any current or future media, including reprinting/republishing this material for advertising or promotional purposes, creating new collective works, for resale or redistribution to servers or lists, or reuse of any copyrighted component of this work in other works. 


\title{
ANFIS to Estimate Damping Coefficient from EMG to Optimize the Interaction Force
}

\author{
Tanvir Anwar, Adel Al Jumaily, Member, IEEE
}

\begin{abstract}
Lower Limb Robotic Rehabilitation device exhibit a great prospect in the rehabilitation of impaired limb, yet it has not been widely applied to clinical rehabilitation. This is mostly due to the insufficient bidirectional information interaction between exoskeleton and patient. In the shared control at the interaction point, it is very important that the deficiency of impaired lower limb in sharing the knee joint dynamics (Capturing of the intended action of the patient) is extracted beforehand to estimate as to how much assistance the robotic exoskeleton would provide. The intended action data that can be extracted from EMG signal may include the intended posture, intended torque, intended knee joint angle, intended knee joint torque and impedance parameter. In this paper, an application of Adaptive Network Based Fuzzy Inference System (ANFIS) has been proposed for proprioceptive feedback on the status of the interaction force at the patient robotic exoskeleton interaction point. ANFIS has been used to model the relationship between input and output. Interaction forces, rate of change in surface electromyography (EMG) signal are two inputs to ANFIS model and impedance parameters damping coefficients (also stiffness) is output. Impedance control law has damping as one of the tuning parameter. The resultant total torque is calculated from this law. The proposed model is able to estimate damping and demonstrate decent accuracy in modulating the knee joint dynamics to minimize the interaction force at the Patient Exoskeleton interaction point.
\end{abstract}

\section{INTRODUCTION}

The conventional Robotic Rehabilitation Device (RRD) is in the pattern of industrial robot which still behaves like master-slave manner. One of the main objectives of a RRD is to obtain a smooth human machine interaction in different phases of gait cycle at the interaction point by considering patient-exoskeleton interaction is bidirectional rather than unidirectional. A design of an effective wearable exoskeleton controller to achieve bidirectional interaction is possible where minimum interaction force is experienced, since patient now become an active element of a closed loop control system [1]. In human body the motor system is a typical closed loop control system where brain and somatic nervous system (SNS) are the controllers that generates necessary signal for muscle which is the actuator and also with a proprioception feedback channel, which is the biological basis of human motion stability. Half of closed loop control system is the human proprioception which is a distinct sensory modality that provides internal feedback solely on the status of the body internally. On the other hand

\footnotetext{
*Research supported by Centre of Health of University of Technology, Sydney

Author Tanvir Anwar is doing PHD at University of Technology, Sydney, Australia. (E-mail: tanvir.anwar@student.uts.edu.au).

Author, Professor Adel Al Jumaily is the associate professor in University of Technology, Sydney. (adel-al.jumaily@uts.edu.au)
}

in the exoskeleton device, Robot is the controller and generate necessary signal for the actuator (DC motor, hydraulic actuator). EMG signal, exoskeleton knee Joint angle, torque, patient-exoskeleton interaction forces are proprioceptive feedback to the robot controller about the status of exoskeleton performance while interacting with human limb. When patient's brain is affected due to stroke or other injury, then brain cannot generate necessary signal for limb movement or perceive the motion status of their legs. Then Robotic Rehabilitation Device shares most of the joint activities and help patient perform the required movements. Being inspired by the closed loop control system of human, a bidirectional human machine interface has been proposed in this paper. As a result of robotic therapy, the brain will be able to generate necessary EMG signal once again responsible for lower limb movement in the long run. This process is called plasticity. Whole process of brain plasticity has to undergo different phases of rehabilitation. Knee and hip joint of lower limb robotic rehabilitation device requires a certain set of joint kinematics and dynamics for each phase of rehabilitation. Dynamic parameters (damping, stiffness, inertia) associated with EMG are always changing and it is important that these parameters are captured from EMG to produce suitable control signal to generate desired knee joint kinematics and dynamics [2].

\section{DATA ACQUISITION}

To boost the data acquisition accuracy, number of electrodes per muscles, the location of the electrodes in the muscle, selection of filters and various features has been used. Two sets of muscles are involved for extension and flexion respectively. 1st set is called Quardriceps which consists of Rectus Femoris, Vastusmedialis and vastuslateralis. This set is responsible for extension. The $2 \mathrm{nd}$ set of muscle is called Hamstrings and it consists of bicep femoris, semitendinosus and semimembranosus. This set is responsible for flexor movement. For knee joint torque estimation, Rectus femoris and Vastusmedialis are selected to observe the effect of load on extension muscle. Bicep femoris and semitendinosus are selected to observe the effect of load on flexion muscle. These muscles will assist us estimate various knee joint dynamic parameters like joint torque, joint angle and changes in the rate of sEMG signal to estimate the stiffness, damping parameters of vitual impedance model to control the exoskeleton knee joint dynamics. sEMG measuring device called flexiComp from Thought Technologyis used to record EMG data at a sampling rate of $2048 \mathrm{~Hz}$ of these muscles simultaneously. The figure 2 shows the location of the muscles respectively for flexion and extension. 


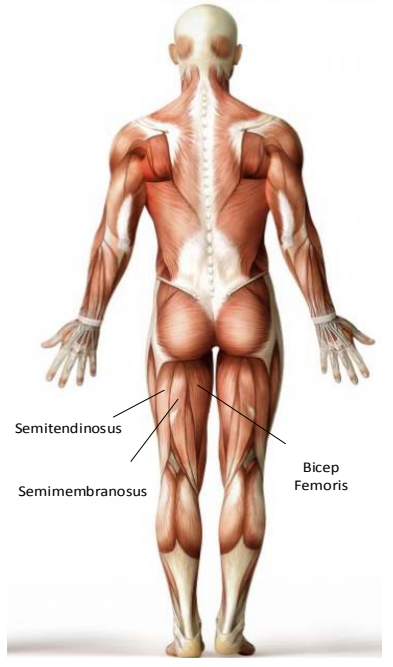

Flexion Muscle

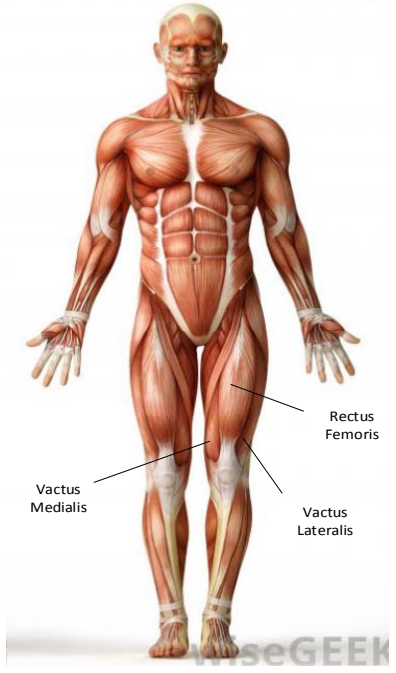

Extensor Muscle
Figure 1. Flexion and Extension Muscle

The exoskeleton knee joint control can be categorized into two major areas of control. The first type of control is the extraction of intended knee joint dynamics (set point or reference for the low level controller). The second type of control becomes necessary when there is inconsistency or discrepancy in the estimation of knee joint angle or knee joint torque. Due to this there is a high degree of interaction force is generated at the patient exoskeleton interaction point which is not desired in a wearable exoskeleton suit that may put patient in uncomfortable situation. So a robotic proprioceptive feedback system is desired to update the knee joint dynamic (knee joint angle, knee joint torque, knee joint velocity and acceleration) from the variables (interaction force, sEMG signal)' associated with patient -Exoskeleton (the controlled object) interaction point that produces a new trajectory to minimize the interaction force. ANFIS has been used as inverse dynamics (Propriceptive Feedback) to intelligently decide as to how much robot will share of the total control sharing at the patient-exoskeleton interaction point. There are four force sensors mounted on the link rotating about an exoskeleton joint sensing the interaction force. The Gyroscope is used to sense the knee joint angle of flexion and extension. Electromyography (EMG) signals are formed by the potentials generated by electrically activated muscle cells, which are $30-100 \mathrm{~ms}$ prior to body motions. So if it is compared with angle and force information, biological EMG signals are better because they can reflect upon the human motion intention in advance and is used as feedback also [2].

\section{SIGNAL PROCESSING}

It is very prominent that original EMG signals are very contaminated during signal acquisition. These noise signals may come from inherent noise in electronic equipment such as industrial frequency interference, DC bias and baseline noise. Motion artifact which is mainly caused by electrode interface and electrode cable will also cause irregularities in sEMG data. Firing rate of the motor units and the firing frequency region $0-20 \mathrm{~Hz}$ also affect the EMG signals. So the removal of the noise is very important. The power density spectra of the EMG contains most of its power in the frequency range of $5-500 \mathrm{~Hz}$ at the extremes, so the signal over the high cut off frequency $500 \mathrm{~Hz}$ should be eliminated. After the above discussion, a notch filter with $50 \mathrm{~Hz}$ and a band pass filter between $20 \mathrm{~Hz}$ to $450 \mathrm{~Hz}$ with a forth order recursive Butterworth filter $(30 \mathrm{~Hz})$ are used to remove the movement artifact.

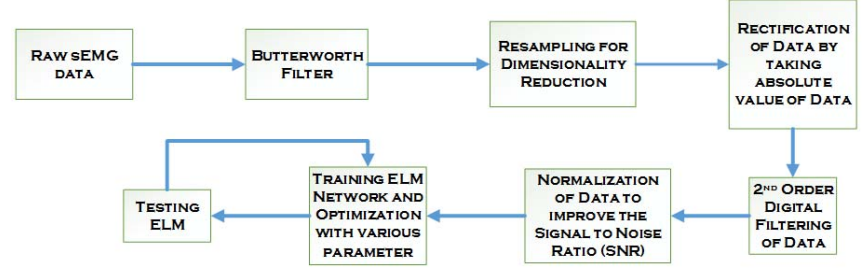

Figure 2. Data Processing to train the ANFIS

Re-sampling, rectification, normalization, feature extraction, feature reduction are various steps towards preparation of an ideal data set ready to be used for training ANFIS or any other intelligent non- linear model.

\section{PROPOSED MODEL}

Figure 3 shows the closed loop adaptive control schematics. The schematic consists of task level, low level and high level controller.

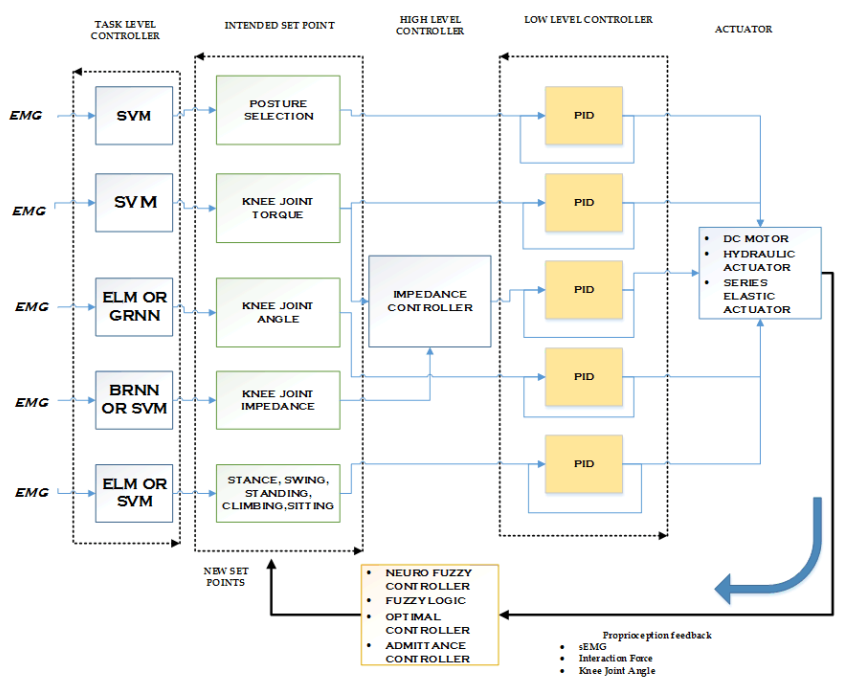

Figure 3. Bidirectional Robotic Rehabilitation Controller[4]

\section{A. The task level}

In this level EMG signal is mapped to intended kinematics and dynamics. These mappings are necessary because EMG and knee joint parameter relationship is highly non-linear and very difficult to model. Extension and Flexion postures are classified with Support Vector machine (SVM). Postures are classified without any need for features of EMG signal due to on/off nature of flexion and extension muscle ( When flexion muscle is active, then extension muscle is inactive and vice versa). Knee Joint angle are estimated with Extreme Machine Learning (ELM, regression type) and Generalized Regression Neural Network (GRNN). sEMG data has been used with wavelet feature for GRNN to 
estimate knee joint angle of extension and flexion. SVM is used to estimate knee joint torque with "Radial Basis Function" as kernel and the parameters of the kernel are used to optimize the performance of the SVM model to estimate knee joint torque.

\section{B. Low Level Control}

PID controllers have been used in low level to execute the reference or adaptive trajectory (desired posture, position, velocity and acceleration) [3].

\section{High Level Control}

In the manipulation of interaction force at exoskeletonpatient interaction point, there are two approaches. If the environment is static (i.e. no muscle activity), then a fixed exoskeleton knee joint dynamic will interact with environment (muscle) with a fixed interaction force (task level control is enough in this case). But if the environment (muscle activity) is changing with respect to time then exoskeleton needs to interact with the environment (muscle) adaptively. So an exoskeleton knee joint is desired which is like spring, mass and damper type of joint rather than rigid joint to interact with a dynamic environment. A virtual impedance controller (control low extracted from spring, mass, damper model) has been used to generate the effect of impedance at the knee joint. The virtual impedance model controls the desired torque of the knee joint by tuning stiffness, damping and inertia parameter of the control law. This is how the property of the exoskeleton knee joint is changed dynamically. ANFIS assists in tuning the knee joint properties. Interaction force and EMG signal are two inputs to the ANFIS to estimate tuning parameter of Damping Coefficient[3].

\section{Proprioceptive Feedback control}

The inverse dynamics of the feedback path of the controller is the proprioceptive feedback of the closed loop controller for updating the status of the patient-exoskeleton interaction. The feedback mechanisms usually include visual, auditory, electrical, and tactile stimulation. An extended proprioception feedback system that features EMG signal, exoskeleton joint torque(techo-generator), angle (encoder or gyroscope) and interaction forces (force sensors)can provide the status that is important for patient and RRD to decide about the new optimized desired set points for the low level controller in a shared control system in a dynamic environment. To achieve an optimized interaction force at the patient-exoskeleton interaction point, it is very important that position, velocity and acceleration states of the exoskeleton are adjusted in the manner that optimized interaction is achieved through ANFIS inverse dynamic model that relates the interaction dynamics at the interaction point to exoskeleton controller (Control law). Through Fuzzy Inference System (rules), EMG signals and interaction force can be related to the damping coefficient of control law.

\section{THE PROPOSED ANFIS ARCHITECTURE}

In this paper an intelligent computational technique has been proposed which will learn patients EMG signal pattern (flexion and extension muscles EMG signal differences) to estimate damping and embed in itself an ability to make intelligent decision. Towards this objective ANFIS has been used to model inverse dynamics which consists of Neural Network and Fuzzy Logic. Neural Network is good at recognizing patterns, but they are not good at explaining how they reach their decisions. The Fuzzy Logic systems, which can reason with imprecise information, are good at explaining their decisions. So a hybrid system where two or more techniques are combined in a manner that they together overcomes the limitations of individual techniques [4].

In Fuzzy model the 1st layer is Fuzzy set. The transition of input EMG and interaction force data from "belonging to a set" to "not belonging to a set" is gradual and this smooth transition is characterized by membership functions. The membership function maps each element of data to a continuous membership value or grade between 0 and 1 .

$$
\begin{gathered}
A=\left\{\left(x, \mu_{\text {interaction force }}(x)\right) \mid x \in X\right\} \\
B=\left\{\left(y, \mu_{\text {rate of } \operatorname{SEMG}}(y)\right) \mid y \in Y\right\}
\end{gathered}
$$

$\mu_{\text {interaction force }}$ and $\mu_{\text {rate of } S E M G}$ are called the membership functions of $\mathrm{x}$ (interaction force) and $\mathrm{y}$ (rate of change in sEMG signal) in A and B respectively. Triangle, Trapezoidal, Gaussian are various membership functions available in Fuzzy Logic Inference System. In this paper there are two independent Fuzzy sets which are Interaction Force and rate of change in sEMG signal intensity. Fuzzy sets are expressed with linguistic expression. In our case Interaction force has been divided into low interaction force: LF, Moderately low interaction force: MLF, Medium Interaction Force: MF, Moderately High Interaction Force: MHF, High Interaction Force: HF. Other fuzzy set of sEMG signal has been divided into Low rate of Change in sEMG signal: LR, Moderately Low Rate: MLR, Medium Rate: MR, Moderately High Rate: MHR, High Rate: HR.

In this paper a Gaussian membership function has been used and is specified by two parameters $\{\sigma, c\}$ as follows:

$$
\operatorname{gaussian}(x ; \sigma, c)=e^{-\left(\frac{x-c}{\sigma}\right)^{2}}
$$

But the model is subject to investigation as to which membership function ensures best accuracy. We may chose different membership function for two different inputs.

Layer 2 is a fixed node labeled, which multiplies the

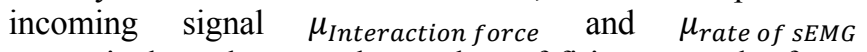
respectively and output the product of firing strength of two membership functions.

$$
O_{2, i}=w_{i}=\mu_{\text {interaction force }}(A) * \mu_{\text {rate of } \operatorname{sEMG}}(B)
$$

In layer 3 , the firing strengths of layer 2 is normalized in the following manner,

$$
O_{3, i}=\overline{w_{i}}=\frac{w_{i}}{w_{1}+w_{2}}, i=1,2
$$


In layer 4 , a fuzzy if-then rule assumes the form as follows: If interaction force $(x)$ is in A and rate of EMG $(y)$ is in B then[5],

$$
O_{4, i}=\overline{w_{i}}\left(P_{i} x+Q_{i} y+r_{i}\right)
$$

Where $\overline{w_{i}}$ is the output of layer 3 and $\left\{P_{i}, Q_{i}, r_{i}\right\}$ are the parameter set. The parameters in this set will be referred to as consequent parameters. From the analysis of EMG signal pattern, it is very prominent that EMG intensity changes linearly with respect to time. So a linear equation is the author's choice to represent the changes in output damping coefficient. When EMG pattern changes positively, damping coefficient should change negatively and linearly. The opposite is also true that in case of negative changes in EMG signal rate, damping should change positively and linearly to ensure smooth human-exoskeleton interaction. The justification of rules generated in Fuzzy Inference System or the nature of adaptation has been discussed later in this paper.

Layer 5 is a single node layer. In this layer overall output of all incoming signal are summed up.

$$
O_{5, i}=\sum \bar{w}_{i} f_{i}=\frac{\sum w_{i} f_{i}}{\sum w_{i}}
$$

$f_{i}=P_{i} x+Q_{i} y+r_{i}$. The output in this paper is stiffness or damping coefficient of the virtual impedance controller. Each one of the above 5 layers are respectively one layer in Neural Network. So the Hybrid ANFIS model is to be trained with data set. Data set consists of Interaction force and rate of EMG signal as input vector and desired stiffness or damping as output data vector[6].

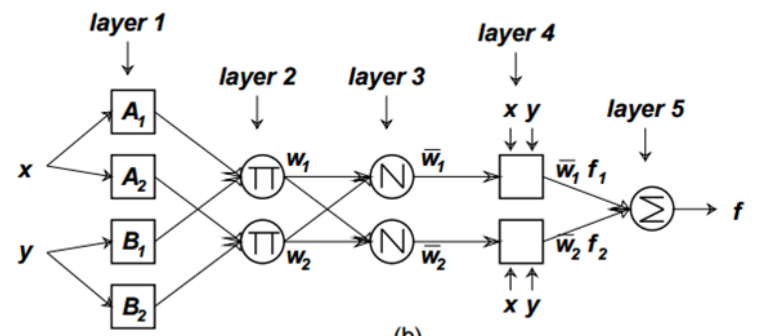

(b)

Figure 4. Neural Network Architecture with Fuzzy embedded

The tuning of the ANFIS parameters are necessary to optimize the performance of the model (minimize the error between desired target output and actual estimated output). We have two sets of parameters. In layer one there are parameters of membership function. Height, width, slope, standard deviation features of the membership function are changed with the tuning of membership function parameters. In this paper Gaussian membership function is used. The $\left\{P_{i}, Q_{i}, r_{i}\right\}$ of the fourth layer also need to be tuned for the optimization of the ANFIS estimation (minimization of error in estimation) of stiffness or damping of impedance controller. The algorithm used for optimization is gradient descent as follows:

$$
\begin{aligned}
& c(t+1)=c(t)-\beta \frac{d E(c, \sigma)}{d c} \\
& \sigma(t+1)=\sigma(t)-\beta \frac{d E(c, \sigma)}{d \sigma}
\end{aligned}
$$

Where E stand for error function which is defined as

$$
\mathrm{E}=\text { actual estimated stiffness }- \text { desired stiffness }
$$

Similarly parameters of consequent function are optimized in the following way

$$
\begin{gathered}
P(t+1)=P(t)-\beta \frac{d E(P, Q, r)}{d P} \\
Q(t+1)=Q(t)-\beta \frac{d E(P, Q, r)}{d Q} \\
r(t+1)=r(t)-\beta \frac{d E(P, Q, r)}{d r}
\end{gathered}
$$

Here $\beta$ is the learning rate during training.

\section{Motivation FOR ADAPTIVE FUZZY RULES GENERATION}

An exoskeleton with an actuation system actively adjusts the joint stiffness with zero, constant and variable impedance. In general we can define the "correct" gait motion in the following three ways, Firstly, tolerate deviation from a given reference trajectory at all joints by the use of a complaint device or controller within a virtual "tunnel". If it is too stiff, patient feels passively moved. If it is too soft, patient is not corrected in space and might have undesired pattern. The overall behavior of the algorithm is that it enforces only small deviations from the reference motion when the patient does not want to change the motion and allows for larger deviations when the patient wants to walk in his/her own way. ANFIS works as high level controller and operates in discrete domain whereas low level controller PID operates in continuous domain. Decision making module of high level controller generates sequence of control actions using its decision rules. These decisions are based on online data collected from various sensors (position, velocity, interaction force, sEMG signal etc.). In this paper is inverse dynamics of the closed loop control system is established with ANFIS. ANFIS has been modeled based on the knowledge of an expert therapist who has played a very important role in designing the Fuzzy System rules. The therapist has recommended a decision rule which prescribes when there is only little effort detected by the force sensors, then the controlled impedance (stiffness, damping, and inertia) is set high in order to enforce a motion that is close to the desired reference motion. The impedance magnitude is reduced as soon as an increased patient effort is detected. With lower impedance magnitude greater deviations from the reference motion are possible, and motion has to be generated to a greater extent by muscular forces rather by the robot. Consequently, the robot assistance and reference motion enforcement is reduced.

Another issue to be addressed to generate rules for ANFIS inverse dynamics is the stability of the robotic exoskeleton. The transient response of the output switches from over damping to critical damping as well as critical damping to under damping or vice versa. An ideal transient response is the one that avoid high inertia because it increases the overshoot. Overshoot increases vibration which is not desired at all. An ideal case avoids too much damping also because it generates rigidity and so does stiffness. The reference torque is taken $20 \mathrm{~N}-\mathrm{m}$ which is normal walking torque of a healthy person. So this torque is the reference the robot sharing controller will work around[7]. 
To make our inverse dynamic system more robust we chose to use EMG signal also as one other input to ANFIS. There is an indication that velocity of the knee is proportional to EMG difference between the extension and flexor muscle performing an ideal damper.

$$
\tilde{x}_{i}(t)=B_{i}\left(\dot{q}_{c i}-\dot{q}_{r i}\right)
$$

Here $B_{i}$ is the damping coefficient of the $i t h$ joint which is a positive constant. $\dot{q}_{r i}$ and $\dot{q}_{c i}$ are respectively the reference and command velocities of the ith joint.

$$
\tilde{x}_{i}(t)=\left(\tilde{x}_{e i}(t)-\tilde{x}_{f i}(t)\right)
$$

Here $\tilde{x}_{e i}$ is the normalized sEMG of extensor muscle of the $i$ th joint at time t. $\tilde{x}_{f i}$ is the normalized sEMG of flexor muscle at time t. Knee joint flexion-extension of lower limb is mostly controlled by the coordinated contraction of a pair of muscles, with flexor muscle contracting to implement the flexion and extensor muscle contracting to implement the extension. Therefore, the voluntary effort of the knee joint is defined as the EMG difference of extensor and flexor muscles, which can be used as the control signal of the robotic exoskeleton. When $\tilde{x}_{2}(t)>0$, the contraction of vastus lateralis muscle (extensor muscle) plays the dominant role. As a result, the knee performs an extension. When $\tilde{x}_{2}(t)<0$, the knee performs a flexion since bicep femoris muscle contracts while vastus lateralis muscle relaxes. When $\tilde{x}_{2}=0$, both the extensor and flexor muscles are in relaxation, and consequently the knee maintains still. So $B_{i}$ of the knee joint will very for high variation into the sEMG differences with respect to time. Stiffness of the exoskeleton will be inversely proportional to EMG differences of flexion and extension muscles.

\section{ISSUS THAT DETECTS FUZZY RULES AND METHODOLOGY}

There are two sets of information provided into the Fuzzy inference system that is embedded into the Neural Network of a hybrid ANFIS model. These are respectively interaction force, changes in difference of EMG signal between flexor and extensor. There are two direction of the actuator due to flexion and extension. By our convention, the direction of flexion is clockwise and the direction of extension is anti clockwise. In either way the difference between the EMG of flexion and EMG of extension will increase because the flexion-extension muscle works pretty much like on-off fashion. But by the above convention the increase during flexion is considered positive increase and opposite otherwise (negative increase). The nature of increase in EMG may differ due to the type of postures we are dealing with. In this paper we are concerned with difference in two EMG signals of flexor and extensor muscle because this difference defines the nature of damping. Damping is that phenomena that signifies the amount of energy that is being drained out from the actuator control signal. Low damping means high joint torques and high damping means low joint torques. In case of higher degree of difference between EMG signals of flexion and extension muscles, lower degree of damping is desired. The opposite is true in case of lower degree of difference between EMG signals of two respective posture muscles.
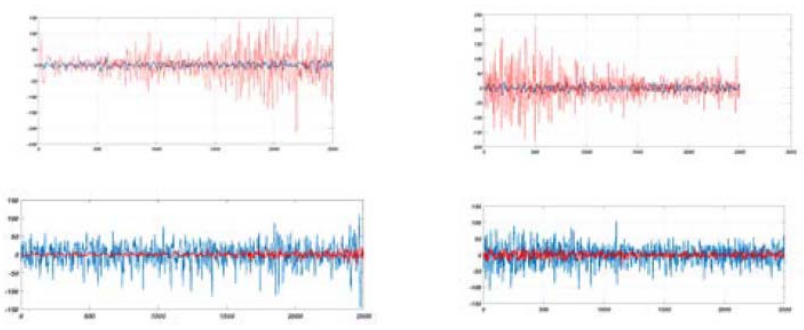

Figure 5. Ch1(Red Color) is high during flexion and Ch2 (blue Color) is high during Extension.
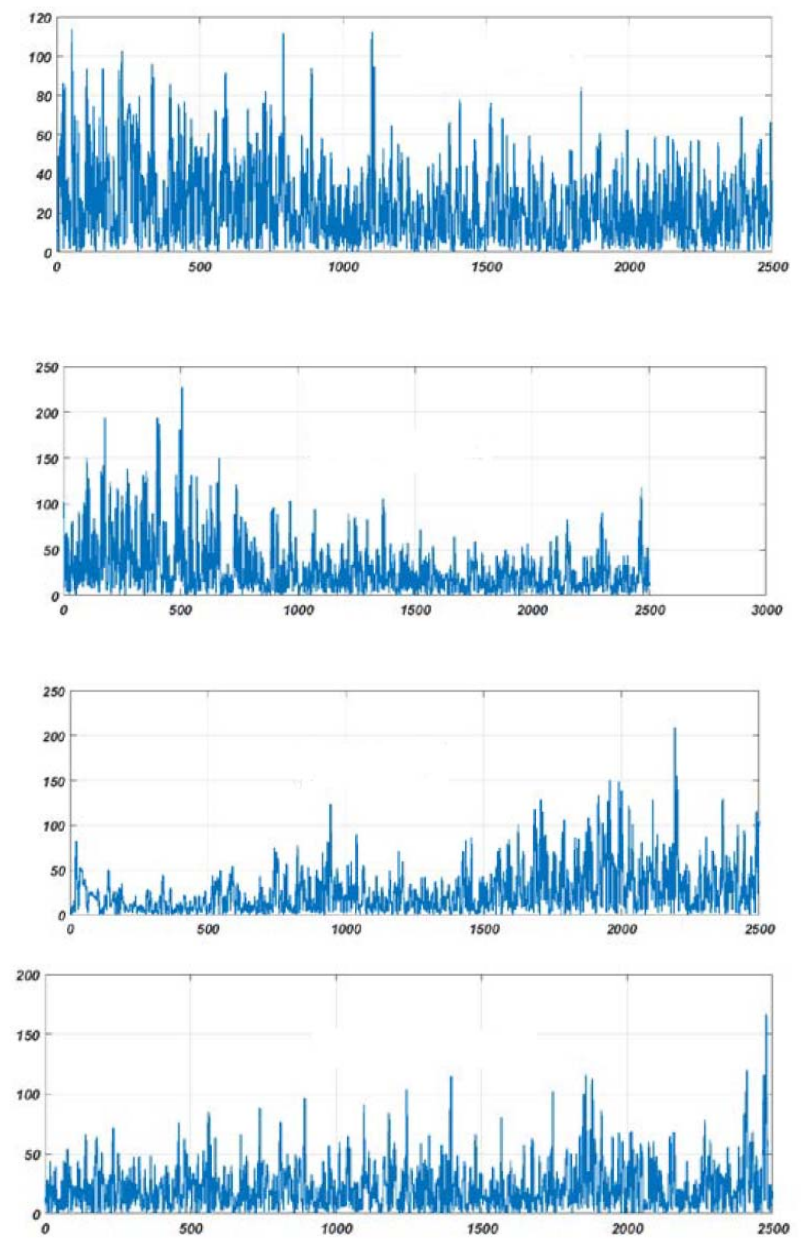

Figure 6. Four EMG patterns of flexion and extension muscles in complete gait cycle.

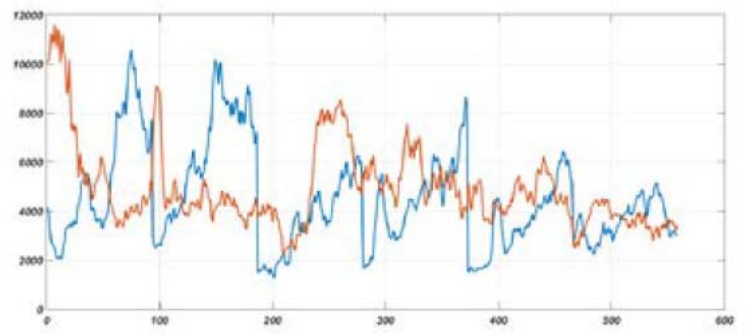



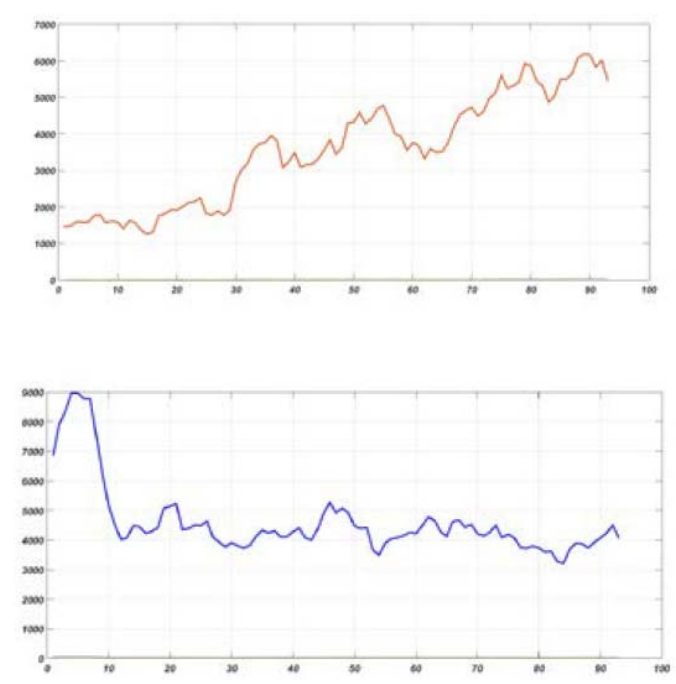

Figure 7. EMG signal difference of (a) six cycles, (b) during flexion and (c) during extension movement.

TABLE I. RULE DATABASE OF FUZZY CONTROLLER

\begin{tabular}{|l|l|c|}
\hline \multicolumn{3}{|c|}{ Fuzzy Inference Model } \\
\hline $\begin{array}{c}\text { Antecedent rule of } \\
\text { interaction force }\end{array}$ & $\begin{array}{c}\text { Antecedent rule of } \\
\text { rate of sEMG signal }\end{array}$ & $\begin{array}{c}\text { Consequence rule of } \\
\text { impedance (damping or } \\
\text { stiffness) }\end{array}$ \\
\hline $\begin{array}{l}\text { If Interaction Force } \\
\text { is Low }\end{array}$ & If sEMG rate is Low & 3.5 \\
\hline $\begin{array}{l}\text { If Interaction Force } \\
\text { is Medium }\end{array}$ & $\begin{array}{l}\text { If sEMG rate is } \\
\text { Medium }\end{array}$ & 2.5 \\
\hline $\begin{array}{l}\text { If Interaction Force } \\
\text { is High }\end{array}$ & $\begin{array}{l}\text { If sEMG rate is } \\
\text { High }\end{array}$ & 1.5 \\
\hline
\end{tabular}
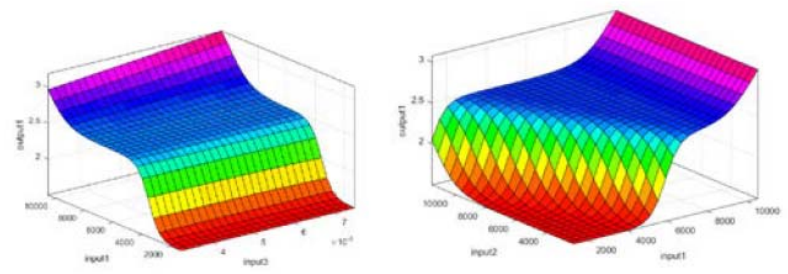

Figure 8. Three dimensional surface plot of effect of Interaction force $(\Delta \mathrm{F})$ and rate of EMG $(\Delta \mathrm{E})$ to output(stiffness)

So a different degrees of freedom tunnel is ensured for smooth human machine interaction. With low impedance higher degree of deviation and with high impedance low deviation is expected

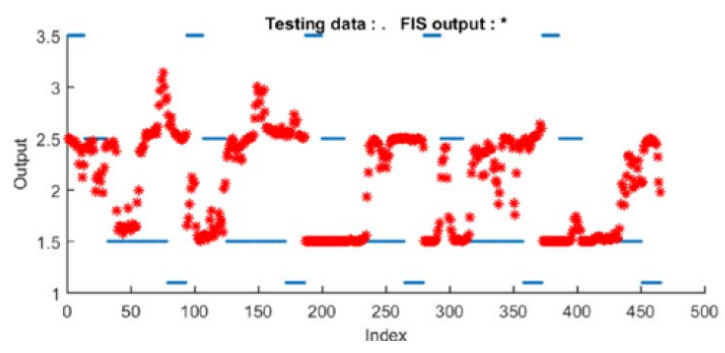

Figure 9. ANFIS performance to estimate stiffness

\section{DATA ANALYSIS}

ANFIS is trained with data set with a data set of $298 \times 3$ with two input vector of interaction force and rate of EMG signal features and one output vector of damping coefficient. The range of interaction is from $0.0025 \mathrm{~N}-\mathrm{m}$ to $0.0085 \mathrm{~N}-\mathrm{m}$. The range of EMG is from 0 to 12000. Only RMS feature of EMG vector is used to generate EMG patterns for ANFIS. The range of damping coefficient is from 0 to 4 . The input vectors are input to Gaussian Membership Function and output vector is taken from linear Membership Function. ANFIS is trained with data set and performance of ANFIS as estimator of damping coefficient is optimized through back propagation through tuning of $\mathrm{C}, \sigma$ of Gaussian membership function and tuning of $\mathrm{P}, \mathrm{Q}, \mathrm{r}$ of output linear membership function. Figure 9 shows interesting result of the performance where estimated damping coefficient (Red color) follows closely the desired damping coefficient (Green color).

\section{CONCLUSION}

A model was desired to learn or capture the EMG damping pattern of different subjects with different severity levels of stroke, EMG patterns at different phase of rehabilitation or EMG patterns of different age groups and make intelligent decisions at the same time as to how much damping is ideal for the robot rehabilitation device in the shared controlled system. Proposed ANFIS model have served the purpose.

\section{ACKNOWLEDGMENT}

I acknowledge the contribution of my supervisor professor Adel Al Jumaily.

\section{REFERENCES}

[1] Y.H. Yin, Y.J.Fan, and L.D.Xu, "EMG and ERP-integrated humanmachine interface between the paralyzed and rehabilitation exoskeleton," Information Technology in Biomedicine, IEEE Transaction on, vol. 16,pp. 542-549,2012

[2] S. N. i. Sidek and A. J. H. Mohideen, "Mapping of EMG signal to hand grip force at varying wrist angles," in Biomedical Engineering and Sciences (IECBES), 2012 IEEE EMBS Conference on, 2012,pp. 648-653.

[3] I. Guler and E. D. Ubeyli, "Adaptive neuro-fuzzy inference system for classification of EEG signals using wavelet coefficients," Journal of neuroscience methods, vol. 148, pp.1130121, 2005.

[4] P. Parker, K. Englehart, and B. Hudgins, "Myoelectric signal processing for control of powered limb prostheses, "Journal of electromyography and kinesiology, vol. 16, pp.541-548, 2006.

[5] E. D. Ubeyli, "Adaptive neuro-fuzzy interface system for classification of ECG signals using Lyapunov exponents," Computer methods and programs in biomedicine, vol. 93, pp. 313-321, 2009.

[6] J. Hu, Z-G. Hou, L. Peng, and N. Gu, "sEMG-Based Single-Joint Active Training with iLeg-A Horizontal Exoskeleton for Lower Limb Rehabilitation," in Neural Information Processing, 2004, pp.535-542.

[7] T. Takagi and M. Sugeno, "Fuzzy identification of systems and its applications to modeling and control," Systems, Man and Cybernetics, IEEE Transactions on, pp.116-132, 1985. 